\title{
Optimization of Multiresonant Wireless Power Transfer Network Based on Generalized Coupled Matrix
}

\author{
Qiang Zhao and Anna Wang \\ College of Information Science and Engineering, Northeastern University, Shenyang, Liaoning 110819, China \\ Correspondence should be addressed to Qiang Zhao; lnshzq@126.com
}

Received 21 April 2017; Accepted 21 May 2017; Published 21 June 2017

Academic Editor: Wanquan Liu

Copyright (C) 2017 Qiang Zhao and Anna Wang. This is an open access article distributed under the Creative Commons Attribution License, which permits unrestricted use, distribution, and reproduction in any medium, provided the original work is properly cited.

\begin{abstract}
Magnetic coupling resonant wireless power transfer network (MCRWPTN) system can realize wireless power transfer for some electrical equipment real-time and high efficiency in a certain spatial scale, which resolves the contradiction between power transfer efficiency and the power transfer distance of the wireless power transfer. A fully coupled resonant energy transfer model for multirelay coils and ports is established. A dynamic adaptive impedance matching control based on fully coupling matrix and particle swarm optimization algorithm based on annealing is developed for the MCRWPTN. Furthermore, as an example, the network which has twenty nodes is analyzed, and the best transmission coefficient which has the highest power transfer efficiency is found using the optimization algorithm, and the coupling constraints are considered simultaneously. Finally, the effectiveness of the proposed method is proved by the simulation results.
\end{abstract}

\section{Introduction}

Since wireless power transfer was firstly proposed by Nikola Tesla 100 years ago [1], much attempts have been devoted to this field. But these attempts did not get much breakthrough for several decades. Until 2006, a MIT research team found that efficient mid-range wireless power transfer could be achieved by utilizing near field magnetic coupling between two identical resonators $[2,3]$. This progress excited renewed interest in this subject.

The magnetic coupling resonant wireless power transmission network (MCRWPTN) is to achieve wireless energy transmission through the resonant coils. In order to improve the transmission efficiency and power and adapt to the needs of the system, the MCRWPT has a variety of advanced multiresonator transmission structures, including relay resonator structure, multitransmit coil, or transmit coil array structure, multireceive coil, or multiple load structure. With the increase of the complexity of the multiresonator structure, a series of new design problems have been introduced in the MCRWPT system, which is a hot research direction in the field of MCRWPT technology.

From the mutual inductance formula, the transmission distance can be increased by increasing the size of the coil, but it is not feasible to apply the method to the limit of the coil geometry. Reducing the load of the transmitter and receiver can also increase the transmission distance of [4], but the actual application of the load cannot be arbitrarily changed; this method also has a lot of limitations. The increase of relay resonator is an effective way to improve the transmission distance.

The concept of relay coil was put forward in [5] based on the induction of electromagnetically induced transparency in quantum interference. By using the coupled mode theory, the energy transfer efficiency of the system was greatly improved by adding a coil with the same resonant frequency between the transmitting coil and the receiving coil. Further study in [6-10] showed that the higher transmission efficiency and power level can be achieved at a larger distance by increasing the relay resonator.

The effect of the addition of 1 relay coil on the transmission efficiency of the MCRWPT system with four coils was studied in [7], and the coaxial relay coil placed on the system to enhance the transmission efficiency was greater, but the vertical display had wider practical application prospects. The [8] proposed MCRWPT array resonator based on $N$ resonator through the relay placed level could achieve $N$ times the diameter of the resonator of wireless power 
transmission (WPT) distance, when using the 10-array resonator, the efficiency of energy transmission was still close to $85 \%$. An efficient WPT system with a relay coil was designed in [10]. Based on the analysis and simulation of the circuit model, the appropriate operating frequency was selected to achieve a transmission power of $6.6 \mathrm{~kW}$ at a distance of $200 \mathrm{~mm}$ and transmission efficiency was $95.57 \%$.

In the practical application of radio energy transmission, such as charging for mobile electronic devices, there was a large demand for the supply of multiple devices simultaneously. With the development of WPT system, more and more attention had been paid to the research of multireceiving coil and multiple load structure.

The basic transmission characteristics such as transmission efficiency and power of multireceiving coil in [11-13] were studied by using circuit model or coupled mode theory. The research on the multireceiver coil structure in WPT was carried out in [11], which verified the feasibility of transmitting the power to a plurality of receiving coils. In a single transmitting coil to the two receiving coils power supply research in [12], the results show that although the transmission efficiency of each single coil is relatively not high, it overall can achieve higher transmission efficiency of the system. Based on the coupled mode theory, [13] was used to study the transmission of multiple load coils. With the same conclusion as in [12], with the two receiving coils, the overall transmission efficiency of multiple receive coils is higher than that of a single receiver coil, but as the number of receivers increases, the overall efficiency of the system tends to saturate and the efficiency of each individual receive coil becomes very low. In [14], the cross-coupling of multireceiving coils was considered, and the optimal design of multiload reception was realized by impedance matching of the transmitter. The paper [15] presented a novel analysis method using band-pass filter model, which made the circuit model with multireceiving coil system more simple and easier to analyze, but its use conditions were relatively harsh.

In [16], the concept of time-sharing transmission in communication system was used to study the multiload charging in MCRWPT system. It was found that time-sharing wireless transmission can achieve efficient and uniform power transmission. The literature [17] was further extended to $N$ resonant coils. The simulation results showed that when there were $N$ resonant coils in the system and the frequency splitting occurred, there would be $N$ efficiency peak points. When there were odd resonant coils, the system transmission efficiency could reach the peak at the original resonant frequency of the coil, and when the number of resonant coils was even, the transmission efficiency was shifted at the original resonant frequency.

The impedance matching method on the load side can simultaneously realize the compensation of the frequency splitting phenomenon and the control of the power distribution. Considering the limitation of the frequency tracking method, the impedance matching is the main control strategy in the multicoil system. The impedance matching strategy for power allocation was studied in $[15,18,19]$. In the actual system, the coupling cannot be ignored when the number of receiving coils is very close $[15,18]$, considering the cross-coupling between multiple receiving coils. The impedance matching and power division method utilizing impedance inverters only at receiver sides was proposed. The mathematical equations in the proposed method were then generalized for arbitrary number of receivers and arbitrary number of repeaters. The coupling of the driving coil and the multiple receiving coils was studied in [19], and it was pointed out that the coupling will change the optimal impedance matching condition given the design method of matching impedance at this time.

Using the scattering parameters as the objective function of the fully coupling matrix extraction and combining the particle swarm optimization algorithm with simulated annealing technique to optimize impedance matching are the goal of this work. The control strategy must overcome some difficulties such as the highly nonlinear and coupled dynamics more over the dynamic, complex, and unstructured environments which may cause unpredictable disturbances to the impedance matching. So dealing with some states in each step will improve matching speed and accuracy. Using the coupling analysis and extraction condition of fully coupling matrix to design an optimal impedance matching strategy for MCRWPTN, it has been shown that the resulting optimization has excellent performance, as demonstrated by simulations.

The paper is outlined as follows. The model of multirelay MCRWPTN is established, and the coupling relation between multiple coils is analyzed in Section 2. The generalized coupling matrix of the system is extracted and an optimal design method is given in Sections 3 and 4. Simulations using FEKO and MATLAB and experiments are performed to validate the new method in Section 5. Finally, Section 6 presents some conclusions.

\section{Problem Formulation}

Wireless power transfer network (WPTN) is an energycentric network, which includes an exciting coil, a transmitting coil, a relay coil, a receiving coil, and a pickup coil. The generalized coupling model of the single energy transmission link composed of $n$ relay energy nodes in the WPTN network is shown in Figure 1.

An internal resonance network is composed of a transmitting, a relay, and a receiving coil, and the resonant frequency and the structure of the transmitting coil, the relay coil, and the receiving coil are the same. The exciting coil and the pickup coil are connected to the power and load ports, respectively, and form an external resonant network with the internal resonant network. The magnetic coupling of the internal resonant network forms a contactless energy transmission link, and multiple transmission links constitute the entire WPTN.

In Figure $1, C_{p}, L_{p}$, and $R_{p}(p=S, L)$ are power and load port parameters; $C_{i}, L_{i}$, and $R_{i}$ are the parameters of each coupling node. $M_{i p}$ is the mutual inductance between the port and the node; $M_{i, i+1}$ is the mutual inductance between adjacent nodes. 


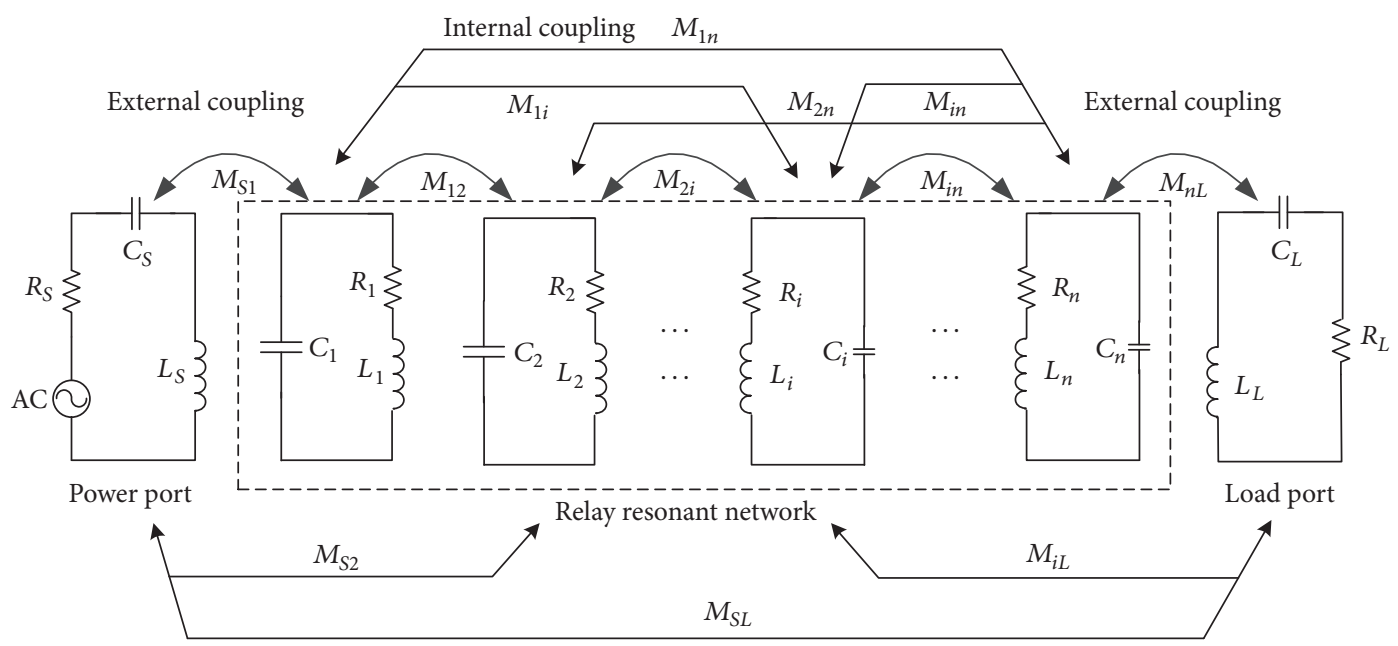

FIGURE 1: Schematic of a wireless power transfer route.

The $Z$ parameter equation of the two-port system can be expressed as

$$
\begin{aligned}
& {[Z] *[I]=j\left(m_{n}-\lambda r-j p\right) *[I]=[V],} \\
& {\left[\begin{array}{ccccc}
Z_{S S} & Z_{S 1} & \cdots & Z_{S n} & Z_{S L} \\
Z_{S 1} & Z_{11} & \cdots & Z_{1 n} & Z_{1 L} \\
\vdots & \vdots & \ddots & \vdots & \vdots \\
Z_{S n} & j Z_{1 n} & \cdots & Z_{n n} & Z_{n L} \\
Z_{S L} & Z_{1 L} & \cdots & Z_{n L} & Z_{L L}
\end{array}\right]\left[\begin{array}{c}
I_{S} \\
I_{1} \\
\vdots \\
I_{n} \\
I_{L}
\end{array}\right]=\left[\begin{array}{c}
V_{s} \\
0 \\
\vdots \\
0 \\
0
\end{array}\right],}
\end{aligned}
$$

where $[Z]$ is the system equivalent impedance matrix and $[m]$ is a fully coupling matrix. For systems with $i(i=1, \ldots, n)$ resonant networks and $p$ ports, the order of the equivalent impedance matrix $[Z]$ is $n+2 . \lambda$ is the normalized frequency factor; $r$ is the unit matrix. $p$ is the port matrix, except for $p_{11}=Z_{S S}$ and $p_{n+2, n+2}=Z_{L L}$ and other elements are zero.

The impedance matrix of the system is shown in (3). Matrix [ $Z]$ is derived from the loop equation formulation of the source and load, external and inner-resonator couplings, and resonator impedances.

$[Z]$

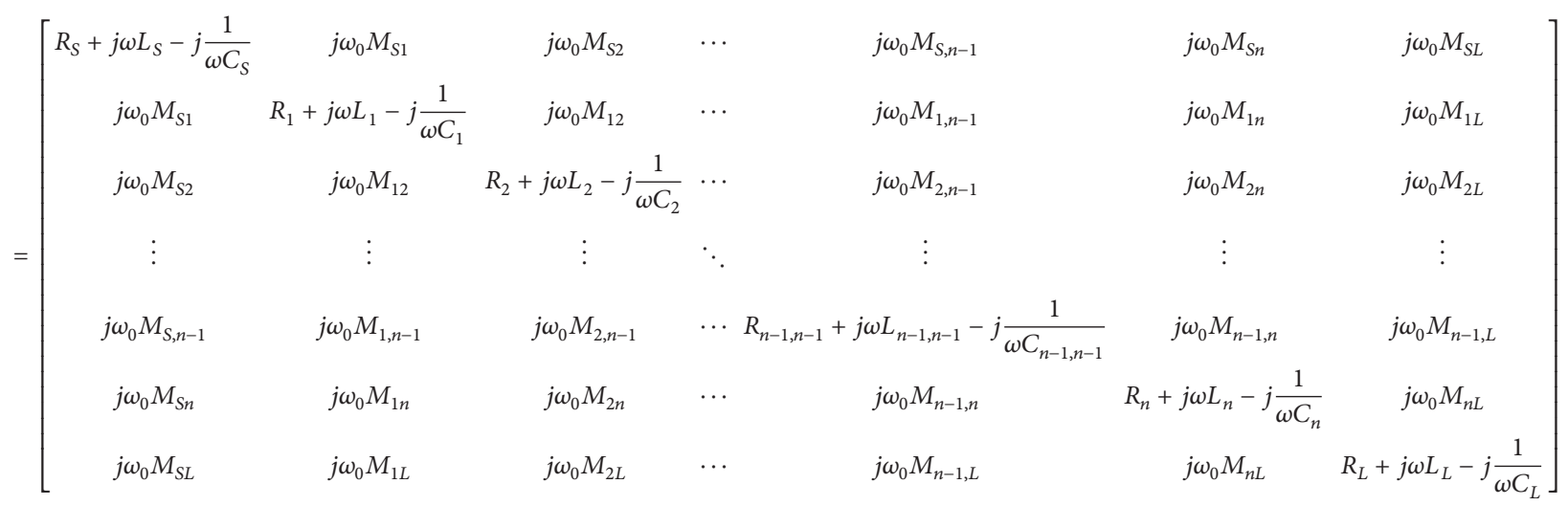

Without loss of generality, we can assume that all nodes are fixed and stationary randomly distributed within the network, and each node has a unique number. All nodes have the same structure and circuit topology, and the coupling 
between nonadjacent nodes is negligible. Each node has the same function and status equivalence as the source node, relay node, and destination node.
The impedance matrix $[Z]$ is normalized as shown in

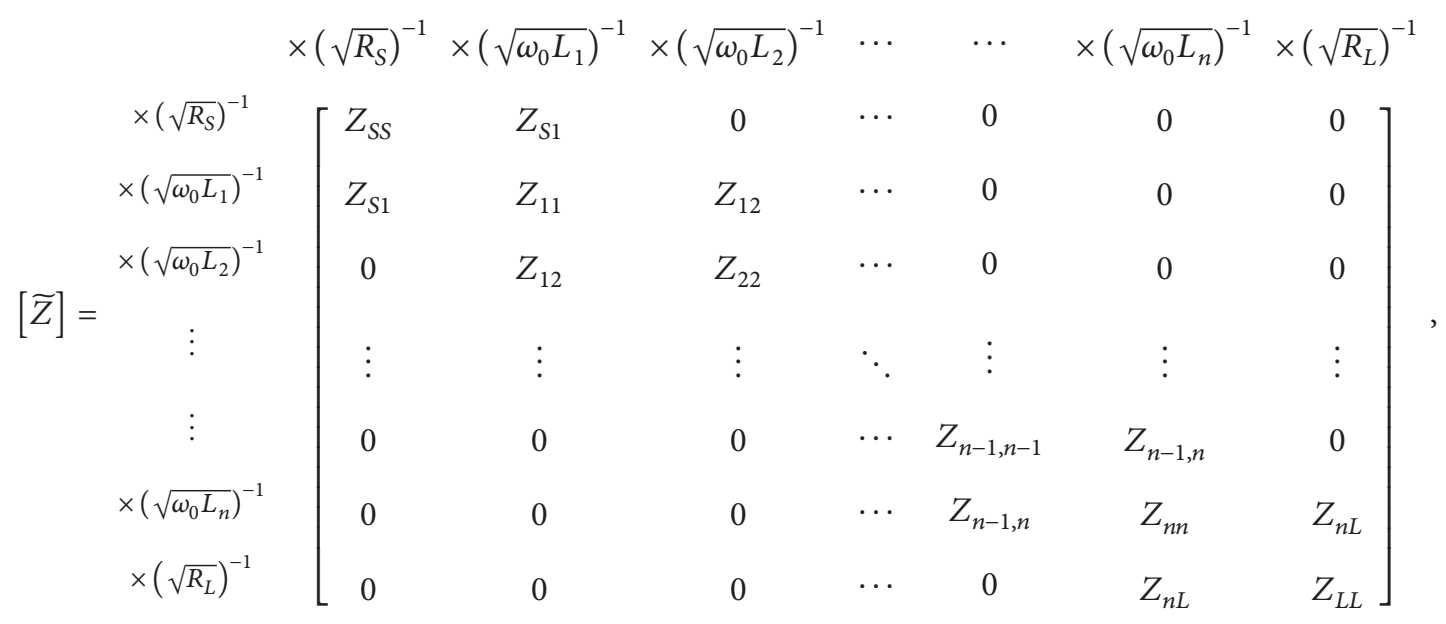

where $1 / \sqrt{R_{p}}$ is an impedance scaling factor $(p=S, L)$, $1 / \sqrt{\omega_{0} L_{i}}(i=1, \ldots, n)$ is a resonator scaling factor, and $\omega_{0}=1 / \sqrt{L_{i} C_{i}}$.
Generalize matrix $[Z]$ such that the resultant matrix can be normalized in terms of couplings and port impedances only. The resultant matrix is the normalized impedance matrix $[\widetilde{Z}]$, as shown in

$$
[\widetilde{Z}]=\left[\begin{array}{ccccccc}
1+j \frac{X_{S}}{R_{S}} & j \frac{\omega M_{S 1}}{\sqrt{\omega_{0} R_{S} L_{1}}} & 0 & \cdots & 0 & 0 & 0 \\
j \frac{\omega M_{S 1}}{\sqrt{\omega_{0} R_{S} L_{1}}} & \frac{1}{Q_{1}}+j \lambda & j \frac{M_{12}}{\sqrt{L_{1} L_{2}}} & \cdots & 0 & 0 & 0 \\
0 & j \frac{M_{12}}{\sqrt{L_{1} L_{2}}} & \frac{1}{Q_{2}}+j \lambda & \cdots & 0 & 0 & 0 \\
\vdots & \vdots & \ddots & \vdots & \vdots & \vdots \\
0 & 0 & \cdots & \frac{1}{Q_{n-1}}+j \lambda & j \frac{M_{n-1, n}}{\sqrt{L_{n-1} L_{n}}} & 0 \\
0 & 0 & 0 & \cdots & j \frac{M_{n-1, n}}{\sqrt{L_{n-1} L_{n}}} & \frac{1}{Q_{n}}+j \lambda & j \frac{\omega M_{n L}}{\sqrt{\omega_{0} L_{n} R_{L}}} \\
0 & 0 & 0 & \cdots & 0 & j \frac{\omega M_{n L}}{\sqrt{\omega_{0} L_{n} R_{L}}} & 1+j \frac{X_{L}}{R_{L}}
\end{array}\right] .
$$

Normalized variables are used in representing the impedance matrix as follows. $Q_{i}=\omega_{0} L_{i} / R_{i}$ is the unloaded $Q$ factor of resonator $i, \lambda=\left(\omega / \omega_{0}-\omega_{0} / \omega\right)$ is the normalized frequency factor, which reflects the relationship between the resonant network and the frequency in the frequency response of MCRWPT. The coupling coefficient of the internal resonator is defined as $k_{i, i+1}=M_{i, i+1} / \sqrt{L_{i} L_{i+1}}$, and the external coupling between resonator $i$ and port $p$ for $i=1, n$ and $p=S, L$ is defined as $E_{S 1}=$ $\omega M_{S 1} / \sqrt{\omega_{0} R_{S} L_{1}}$ and $E_{n L}=\omega M_{n L} / \sqrt{\omega_{0} L_{n} R_{L}}$. The normalized reactance of port $p$ for $p=S, L$ is $x_{S}=X_{S} / R_{S}$ and $x_{L}=X_{L} / R_{L}$. 
This normalized impedance matrix will be referred to as $[\widetilde{Z}]$. Substituting in these variables allows us to make the decomposition of the matrix seen below:

$$
[\widetilde{Z}]=j\left[\begin{array}{ccccccc}
x_{S} & E_{s 1} & 0 & \cdots & 0 & 0 & 0 \\
E_{s 1} & -\frac{j}{Q_{1}} & k_{12} & \cdots & 0 & 0 & 0 \\
0 & k_{12} & -\frac{j}{Q_{2}} & \cdots & 0 & 0 & 0 \\
\vdots & \vdots & \vdots & \ddots & \vdots & \vdots & \vdots \\
0 & 0 & 0 & \cdots & -\frac{j}{Q_{n-1}} & k_{n-1, n} & 0 \\
0 & 0 & 0 & \cdots & k_{n-1, n} & -\frac{j}{Q_{n}} & E_{n L} \\
0 & 0 & 0 & \cdots & 0 & E_{n L} & x_{L}
\end{array}\right]
$$$$
+\left[\begin{array}{ccccccc}
1 & 0 & 0 & \cdots & 0 & 0 & 0 \\
0 & 0 & 0 & \cdots & 0 & 0 & 0 \\
0 & 0 & 0 & \cdots & 0 & 0 & 0 \\
\vdots & \vdots & \vdots & \ddots & \vdots & \vdots & \vdots \\
0 & 0 & 0 & \cdots & 0 & 0 & 0 \\
0 & 0 & 0 & \cdots & 0 & 0 & 0 \\
0 & 0 & 0 & \cdots & 0 & 0 & 1
\end{array}\right]
$$

$$
+j \lambda\left[\begin{array}{ccccccc}
0 & 0 & 0 & \cdots & 0 & 0 & 0 \\
0 & 1 & 0 & \cdots & 0 & 0 & 0 \\
0 & 0 & 1 & \cdots & 0 & 0 & 0 \\
\vdots & \vdots & \vdots & \ddots & \vdots & \vdots & \vdots \\
0 & 0 & 0 & \cdots & 1 & 0 & 0 \\
0 & 0 & 0 & \cdots & 0 & 1 & 0 \\
0 & 0 & 0 & \cdots & 0 & 0 & 0
\end{array}\right] .
$$

Using algebraic manipulation, $[\widetilde{Z}]$ is separated into constitutive components into the form given by

$$
[\widetilde{Z}]=j[m]+j \lambda[r]+[p] .
$$

For the purposes of WPT optimization, the variable $\lambda$ simply reflects the frequency dependency of the MRCWPT frequency response. The unit matrix $[r]$ of the resonant network is $n * n$ identity matrix, the port normalized impedance matrix $[p]$ is zero except for the power and load port elements, the coupling matrix $[m]$ is a reciprocal matrix of $n * n$, and the other nondiagonal elements represent the coupling between the two resonators. If it is zero, the corresponding two resonators are not coupled.

\section{Fully Coupling Matrix Extraction}

From (1) we can see that the current vector can be expressed as

$$
[I]=-j[Z]^{-1}[V] .
$$

Thus, the scattering parameter $S$ of the entire coupling circuit can be expressed as

$$
\begin{aligned}
& S_{21}=2 \sqrt{Z_{S S} Z_{L L}}[I]_{n}=-2 j \sqrt{Z_{S S} Z_{L L}}[Z]_{S L}^{-1}, \\
& S_{11}=1-2 Z_{S S}[I]_{1}=1+2 j Z_{S S}[Z]_{S S}^{-1} .
\end{aligned}
$$

We can get from (7) that

$$
[A]=-j[\widetilde{Z}]=[m]-j[p]+\lambda[r],
$$

where the matrix $[A]$ is built from the coupling matrix $[\mathrm{m}]$, the source matrix $[p]$, and the resonator matrix $[r]$. It can be shown that, from inverting the $[A]$ matrix, the scattering parameters can be found.

$$
\begin{aligned}
& S_{21}=-2 j[A]_{S L}^{-1}, \\
& S_{11}=1+2 j[A]_{S S}^{-1} .
\end{aligned}
$$

The objective function $K$ is uniquely determined by the zero position, the pole position, and the in-band return loss.

$$
\begin{aligned}
K= & \sum_{i=1}^{n}\left|S_{11}\left(\omega_{z i}\right)\right|^{2}+\sum_{i=1}^{p}\left|S_{21}\left(\omega_{p i}\right)\right|^{2} \\
& +\left(\left|S_{11}(\omega=-1)\right|-\frac{\varepsilon}{\sqrt{1+\varepsilon^{2}}}\right)^{2} \\
& +\left(\left|S_{11}(\omega=1)\right|-\frac{\varepsilon}{\sqrt{1+\varepsilon^{2}}}\right)^{2}, \quad(p \leq n),
\end{aligned}
$$

where $\omega_{p i}$ is artificially specified quantity and $\omega_{z i}$ is a function of $\omega_{p i} . \omega_{z i}$ and $\omega_{p i}$ are the zero and pole of the characteristic function $C_{n}(\omega)$, respectively. The latter two are used to determine the in-band return loss. When $K=0$, the target is satisfied and the optimization is finished. Set the following expression:

$$
\begin{aligned}
& C_{n}(\omega)=\frac{1}{2} \frac{G_{n}(\omega)+G_{n}^{\prime}(\omega)}{\prod_{i=1}^{n}\left(1-\omega / \omega_{0}\right)}, \\
& G_{n}(\omega)=\prod_{i=1}^{n}\left[\left(\omega-\frac{1}{\omega_{0}}\right)+\sqrt{\left(\omega^{2}-1\right)\left(1-\frac{1}{\omega_{0}^{2}}\right)}\right], \\
& G_{n}^{\prime}(\omega)=\prod_{i=1}^{n}\left[\left(\omega-\frac{1}{\omega_{0}}\right)-\sqrt{\left(\omega^{2}-1\right)\left(1-\frac{1}{\omega_{0}^{2}}\right)}\right], \\
& G_{n}(\omega)=U_{n}(\omega)+V_{n}(\omega), \\
& G_{n}^{\prime}(\omega)=U_{n}(\omega)-V_{n}(\omega), \\
& U_{n}(\omega)=u_{0}+u_{1} \omega+u_{2} \omega^{2}+\cdots+u_{n} \omega^{n}, \\
& V_{n}(\omega)=\sqrt{\omega^{2}-1}\left(v_{0}+v_{1} \omega+v_{2} \omega^{2}+\cdots+v_{n} \omega^{n}\right) .
\end{aligned}
$$


$G_{1}(\omega)$ can be obtained as substituting the first pole $\omega_{p 1}$ into (14), and the values of $u_{0}, u_{1}, v_{0}, v_{1}$ are obtained by comparing the coefficients, and then determine $U_{1}(\omega), V_{1}(\omega)$.

$$
\begin{aligned}
G_{n}(\omega)= & {\left[U_{n-1}(\omega)+V_{n-1}(\omega)\right] } \\
& \cdot\left[\left(\omega-\frac{1}{\omega_{0}}\right)+\sqrt{\left(\omega^{2}-1\right)\left(1-\frac{1}{\omega_{0}^{2}}\right)}\right] .
\end{aligned}
$$

$G_{2}(\omega)$ can be obtained as substituting the first pole $\omega_{p 2}$, and so on, until the $N$ pole is replaced. From formulas (16) and (17), the molecules of $C_{n}(\omega)$ can be obtained by iteration.

$$
\operatorname{NUM}\left[C_{n}(\omega)\right]=\frac{1}{2}\left[G_{n}(\omega)+G_{n}^{\prime}(\omega)\right]=U_{n}(\omega)
$$

Solving the roots of $U_{n}(\omega)=0$ can obtain the $N$ zeros of the characteristic function. The parameters of the objective function can be determined after substituting the zero point, the pole, and the return loss into (12). The relation between generalized coupling matrix $[m]$ and objective function $K$ can be established by the above derivation, and the optimization model of the coupling matrix extraction is shown in formula (22).

$$
\begin{array}{ll}
\min & K \\
& =\sum_{i=1}^{n}\left|S_{11}\left(\omega_{z i}\right)\right|^{2}+\sum_{i=1}^{p}\left|S_{21}\left(\omega_{p i}\right)\right|^{2} \\
& +\left(\left|S_{11}(\omega=-1)\right|-\frac{\varepsilon}{\sqrt{1+\varepsilon^{2}}}\right)^{2} \\
& +\left(\left|S_{11}(\omega=1)\right|-\frac{\varepsilon}{\sqrt{1+\varepsilon^{2}}}\right)^{2}, \quad(p \leq n) \\
\text { s.t. } \quad C_{n}(\omega)=\frac{1}{2} \frac{G_{n}(\omega)+G_{n}^{\prime}(\omega)}{\prod_{i=1}^{n}\left(1-\omega_{0}\right)} & G_{n}(\omega) \\
= & \prod_{i=1}^{n}\left[\left(\omega-\frac{1}{\omega_{0}}\right)+\sqrt{\left(\omega^{2}-1\right)\left(1-\frac{1}{\omega_{0}^{2}}\right)}\right] \\
\quad & G_{n}^{\prime}(\omega) \\
= & \prod_{i=1}^{n}\left[\left(\omega-\frac{1}{\omega_{0}}\right)-\sqrt{\left(\omega^{2}-1\right)\left(1-\frac{1}{\omega_{0}^{2}}\right)}\right] .
\end{array}
$$

\section{Impedance Matching Optimization}

In order to improve the extraction speed of generalized coupling matrix, a particle swarm optimization algorithm based on simulated annealing is presented. Fitness function can be expressed as follows:

$$
\begin{aligned}
\text { fittness } & =G_{n}(\omega) \\
& =\prod_{i=1}^{n}\left[\left(\omega-\frac{1}{\omega_{0}}\right)+\sqrt{\left(\omega^{2}-1\right)\left(1-\frac{1}{\omega_{0}^{2}}\right)}\right] .
\end{aligned}
$$

Firstly, the initial population is generated randomly, and a new group of individuals is generated by the basic particle swarm optimization algorithm.

$$
\begin{aligned}
V_{i+1}= & w * V_{i}+c_{1} * \operatorname{rand}() *\left(\text { pbest }-x_{i}\right)+c_{2} \\
& * \operatorname{rand}() *\left(\text { gbest }-x_{i}\right) \\
x_{i+1}= & x_{i}+V_{i},
\end{aligned}
$$

where pbest is the best location of the particle itself and gbest is the best location for all particles in the population.

Then, the crossover and the Gaussian mutation operation are carried out independently. Simulated annealing is performed for each individual, and the result is used as an individual in the next generation.

In each evolution, crossover operation selects a specified number of particles into a pool according to crossing probability, which are randomly crossed to produce the same number of children, instead of parents to keep the number of particles unchanged. The position of the child particle is calculated by the weighted sums of the parent particles.

$$
\begin{aligned}
& \operatorname{child}_{1}(x)=p * \operatorname{parent}_{1}(x)+(1-p) * \operatorname{parent}_{2}(x), \\
& \operatorname{child}_{2}(x)=p * \operatorname{parent}_{2}(x)+(1-p) * \operatorname{parent}_{1}(x),
\end{aligned}
$$

where $x$ is the $D$-dimension position vector and child ${ }_{k}(x)$ and parent $_{k}(x), k=1,2$, respectively, are the location of the child or parent particles. $p$ is a uniform random number vector of $D$-dimension, with each component in $[0,1]$ value. The speed of the child particles is given by the following formula:

$$
\begin{aligned}
& \operatorname{child}_{1}(v)=\frac{\operatorname{parent}_{1}(v)+\operatorname{parent}_{2}(v)}{\left|\operatorname{parent}_{1}(v)+\operatorname{parent}_{2}(v)\right|}\left|\operatorname{parent}_{1}(v)\right|, \\
& \operatorname{child}_{2}(v)=\frac{\operatorname{parent}_{1}(v)+\operatorname{parent}_{2}(v)}{\left|\operatorname{parent}_{1}(v)+\operatorname{parent}_{2}(v)\right|}\left|\operatorname{parent}_{2}(v)\right|,
\end{aligned}
$$

where $v$ is the velocity vector of the $D$-dimension.

In each evolution, the specified number of particles is selected by mutation operation according to the mutation probability, which was mutated by Gaussian mutation operator, and the mutated particles are used instead of the original particles.

$$
\text { mutation }(x)=x *(1+\operatorname{Gaussian}(\delta)) .
$$

The implementation of the algorithm is composed of two parts. Firstly, the evolution of the basic particle swarm optimization algorithm (global search) is used to produce a better group, and then the crossover and mutation operations are applied, and the particles are further optimized by simulated annealing (local search). The evolution process is iterated until the termination condition is satisfied. The algorithm flow is as follows:

(1) Initialization parameters.

(2) The population of randomly generated $N$ particles.

(3) Using (24) to manipulate the particles in the population. 
TABLE 1: The system parameters configuration.

\begin{tabular}{lc}
\hline Parameters & Values \\
\hline$R_{S}$ & $50 \Omega$ \\
$R_{L}$ & $50 \Omega$ \\
Port coils & \\
$\quad$ Radius $r$ & $100 \mathrm{~mm}$ \\
$\quad$ Number of turns $N$ & 1 turn \\
Resonant coils & \\
$\quad$ Radius $R$ & $100 \mathrm{~mm}$ \\
$\quad$ Number of turns $N$ & 5 turns \\
$\quad$ Resonant frequency $f_{0}$ & $12.85 \mathrm{MHz}$ \\
$\quad$ Coupling coefficient between resonators $k_{i, i+1}$ & 0.2 \\
$\quad$ Coupling coefficient between nonadjacent & 0 \\
resonators & \\
\hline
\end{tabular}

(4) The population generated by (3) select the particle to form the subpopulation by the crossover probability and carries out the following operations to produce a new species population: from the subpopulation randomly selected individuals $x_{j}$ and $x_{k}$ according to (25) for cross-operation to produce two new individuals $x_{j}^{\prime}, x_{k}^{\prime}$, calculate the adaptive function value $f\left(x_{j}\right), f\left(x_{k}\right), f\left(x_{j}^{\prime}\right), f\left(x_{k}^{\prime}\right)$; if $\min \left\{1, \exp \left(-\left(f\left(x_{j}^{\prime}\right)\right)-\right.\right.$ $\left.\left.f\left(x_{j}^{\prime}\right)\right) / T\right\}>$ random, then $x_{j}^{\prime}$ is a new individual; if $\min \left\{1, \exp \left(-\left(f\left(x_{k}^{\prime}\right)\right)-f\left(x_{k}^{\prime}\right)\right) / T\right\}>$ random, then $x_{k}^{\prime}$ is a new individual, where random is a random number on the $[0,1]$ interval.

(5) The population generated by crossing selects the particle to form the subpopulation by the mutation probability and carries out the following operations to produce a new species population. An individual is selected from the subpopulation to perform a Gaussian mutation operation to produce a new individual $x_{j}^{\prime}$, and calculate the adaptive function value $f\left(x_{j}\right)$ and $f\left(x_{j}^{\prime}\right)$; if $\min \left\{1, \exp \left(-\left(f\left(x_{j}^{\prime}\right)\right)-f\left(x_{j}^{\prime}\right)\right) / T\right\}>$ random, then $x_{j}^{\prime}$ is a new individual.

(6) If the current optimal individual satisfies the convergence condition, the evolution process ends successfully and returns the global optimal solution.

(7) If the number of evolutions is less than the maximum number of times of evolution, the annealing temperature of the population is modified, and then return to step (3).

\section{Simulation Results}

In order to optimize the extraction of generalized coupling matrix parameters fast convergence, the initial value should be set close to the actual value. The parameters of the MCRWPTN system are shown in Table 1 . The optimized and simulated $S$ parameters are shown in Figures $2-4$.

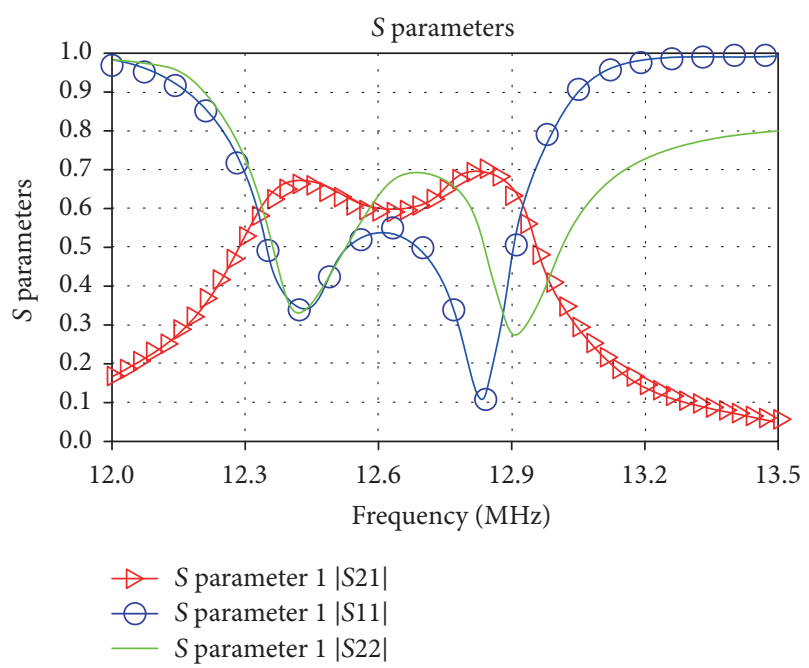

FIGURE 2: The curves of scattering parameters of WPTN.

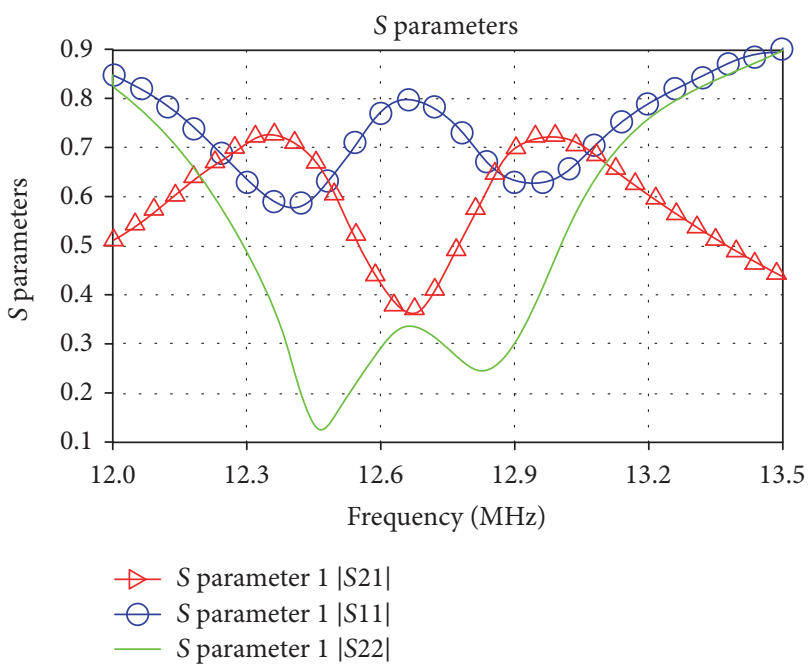

FIGURE 3: The curves of the scattering parameters of external coupling network of power port.

When the number of ports is $p=2$, the number of coupling relay coils is $n=20$, and the system scattering parameter curve is shown in Figures 2-4. These three curves represent the specific distribution of the scattering parameters of the total transmission network and the two transmission subnetworks of the MCRWPTN, respectively, which can reflect the law of the scattering parameters of the system with frequency, where $S_{21}$ is forward transmission coefficient and $S_{11}$ is reflection coefficient of port 1. They can respond to the transmission characteristics of the system, the higher $S_{21}$ is better, and the opposite of $S_{11}$ is as low as possible.

As can be seen from Figure 2, the frequency division of the system has low frequency resonance and high frequency resonance. At the resonant frequency, $S_{21}$ reaches the maximum, and $S_{11}=0.1$ is the smallest. So the maximum power transmission efficiency is obtained. The response curve 


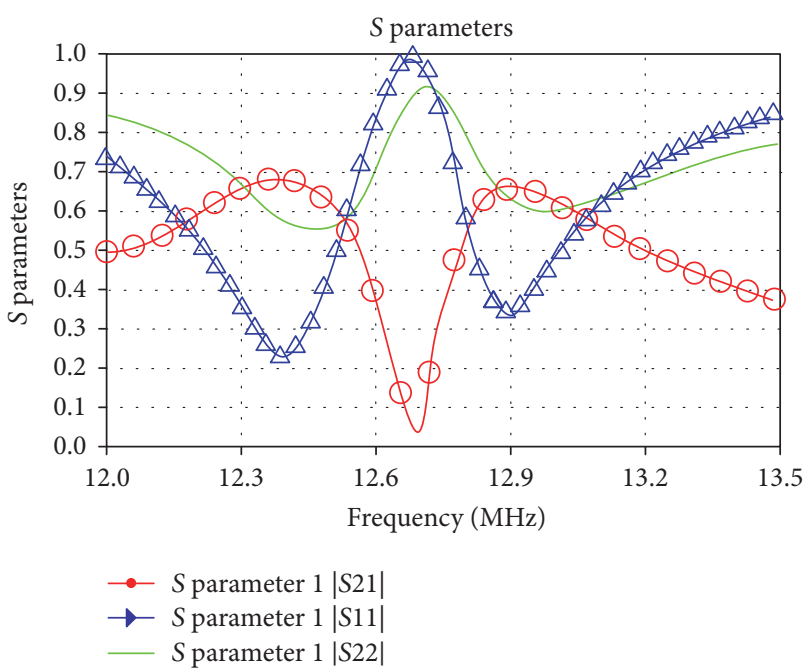

FIGURE 4: The curves of the scattering parameters of external coupling network of load port.

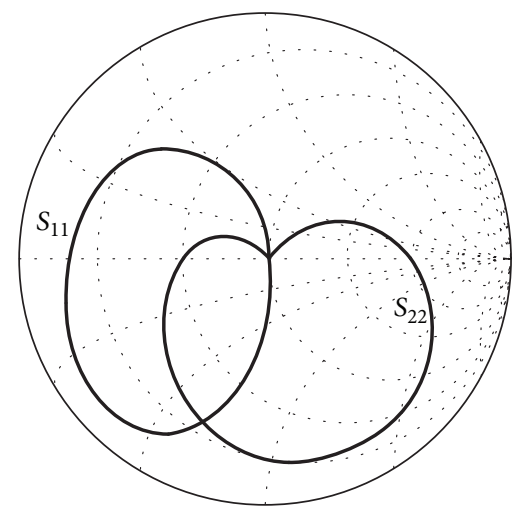

FIGURE 5: Simulation validation of complex load impedance accommodation.

obtained after optimization is in good agreement with the S parameters obtained from the simulation. From Figures 3 and 4 , the existence of external coupling network makes the port impedance match well.

Figure 5 shows the simulated $S_{11}$ and $S_{22}$ responses of the complex load impedance accommodated response measured at the interresonator coupling location $k_{\mathrm{ST}}=0.01$. As shown, the resonance dip of $S_{22}$ response approaches the center of the Smith chart. This indicates that the output impedance of the impedance matching optimized system is the complex conjugate of $Z_{p}=R_{p}+j X_{p}$.

Figure 6 is the modulus value of the reflection coefficient obtained by different algorithms. By comparing the three different algorithms of reflection coefficient magnitude, the particle swarm optimization combined with simulated annealing algorithm is a new type of impedance matching algorithm, and the reflection coefficient tends to zero to achieve impedance matching finally, which is better than the improved particle swarm algorithm in speed.

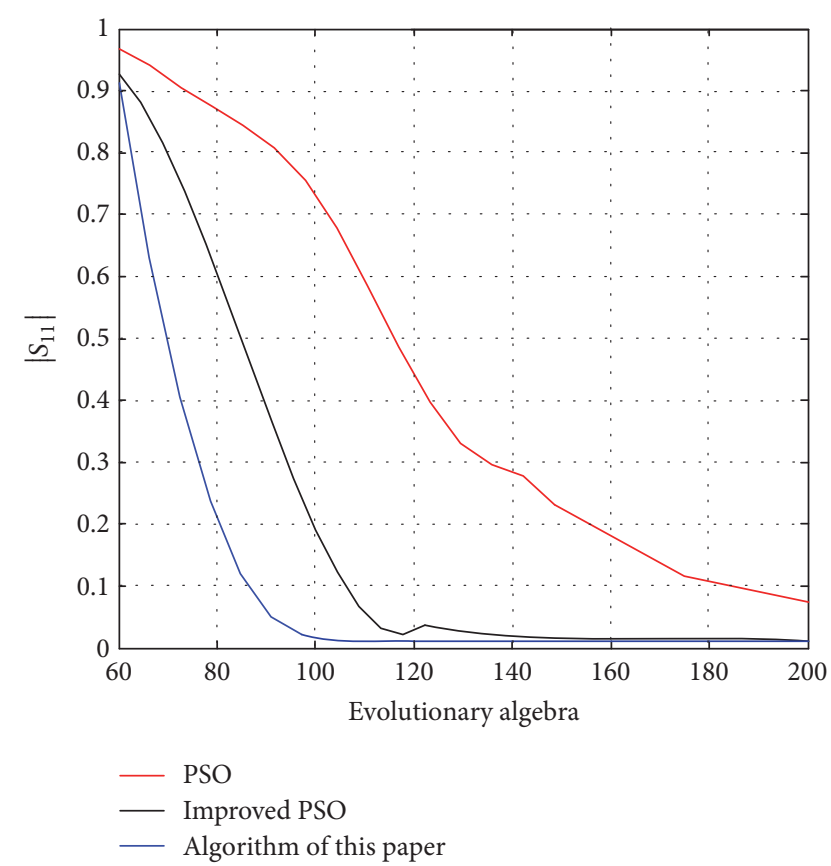

FIGURE 6: The modulus value of the reflection coefficient obtained by different algorithms.

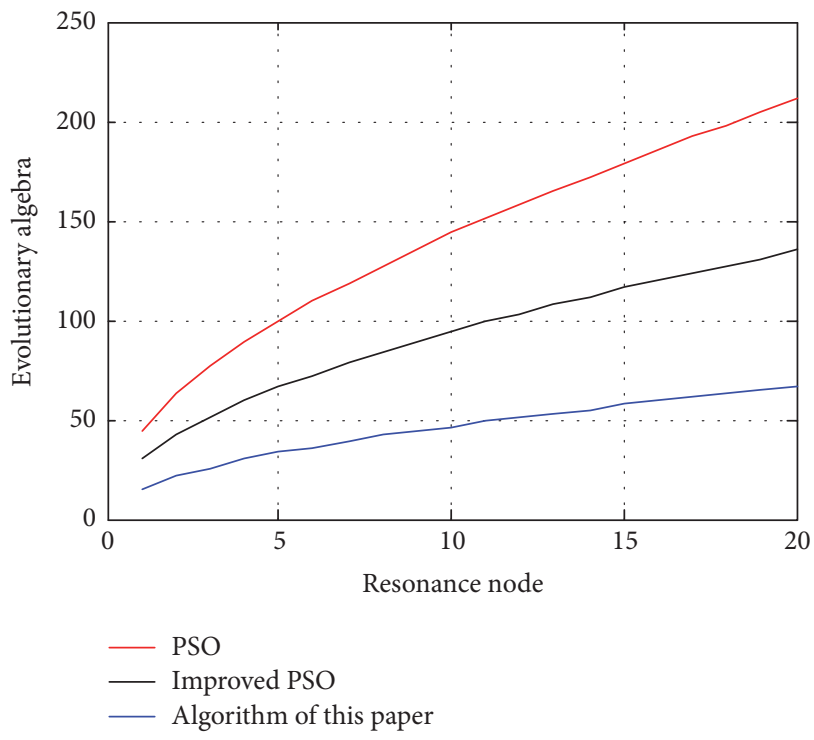

FIGURE 7: Multicoupled node network optimization.

The relationship between the number of coupled nodes and the number of iterations is shown in Figure 7. As the number of nodes increases, the algorithm is superior to other algorithms and is easy to extend to multirelay MCRWPTN.

\section{Conclusion}

A fully coupling matrix for multirelay MCRWPTN is proposed and theory to impedance matching optimization algorithm is developed. This combination will emphasize not 
only the energy transfer but also the matching speed and scalability. The optimization algorithm combines the ability of the simulated annealing algorithm to jump out of the local optimal solution and the global optimization ability of the particle swarm optimization algorithm, which avoids the shortcomings of falling into the local extreme point and improves the algorithm convergence speed and accuracy. Theoretical results are supported by numerical simulations with impedance matching of the proposed fully coupled matrix and optimization algorithm.

\section{Conflicts of Interest}

The authors declare that there are no conflicts of interest regarding the publication of this paper.

\section{References}

[1] A. Kurs, A. Karalis, R. Moffatt, J. D. Joannopoulos, P. Fisher, and M. Soljačić, "Wireless power transfer via strongly coupled magnetic resonances," American Association for the Advancement of Science. Science, vol. 317, no. 5834, pp. 83-86, 2007.

[2] A. K. RamRakhyani, S. Mirabbasi, and M. Chiao, "Design and optimization of resonance-based efficient wireless power delivery systems for biomedical implants," IEEE Transactions on Biomedical Circuits and Systems, vol. 5, no. 1, pp. 48-63, 2011.

[3] A. P. Sample, D. A. Meyer, and J. R. Smith, "Analysis, experimental results, and range adaptation of magnetically coupled resonators for wireless power transfer," IEEE Transactions on Industrial Electronics, vol. 58, no. 2, pp. 544-554, 2011.

[4] M. Fu, T. Zhang, C. Ma, and X. Zhu, "Efficiency and optimal loads analysis for multiple-receiver wireless power transfer systems," IEEE Transactions on Microwave Theory and Techniques, vol. 63, no. 3, pp. 801-812, 2015.

[5] R. E. Hamam, A. Karalis, J. D. Joannopoulos, and M. Soljačić, "Efficient weakly-radiative wireless energy transfer: an EIT-like approach," Annals of Physics, vol. 324, no. 8, pp. 1783-1795, 2009.

[6] M. Meng and M. Kiani, "Design and optimization of ultrasonic wireless power transmission links for millimeter-sized biomedical implants," IEEE Transactions on Biomedical Circuits and Systems, vol. 11, no. 1, pp. 98-107, 2016.

[7] G. Monti, L. Tarricone, and M. Mongiardo, "Two-port network approach for a wireless power transfer link using a cascade of inductively coupled resonators," International Journal of Numerical Modelling: Electronic Networks, Devices and Fields, p. e2234, 2017.

[8] B. Wang, K. Teo, and S. Yamaguchi, "Flexible and mobile nearfield wireless power transfer using an array of resonators," The Institute of Electronics, Information and Communication Engineers, pp. 10-16, 2012.

[9] J. Dai and D. C. Ludois, "A Survey of wireless power transfer and a critical comparison of inductive and capacitive coupling for small gap applications," IEEE Transactions on Power Electronics, vol. 30, no. 11, pp. 6017-6029, 2015.

[10] S. C. Moon and G.-W. Moon, "Wireless power transfer system with an asymmetric four-coil resonator for electric vehicle battery chargers," IEEE Transactions on Power Electronics, vol. 31, no. 10, pp. 6844-6854, 2016.

[11] J. Lee, K. Lee, and D. Cho, "Stability improvement of transmission efficiency based on a relay resonator in a wireless power transfer system," IEEE Transactions on Power Electronics, vol. 32, no. 5, pp. 3297-3300, 2017.

[12] J. Kim, D.-H. Kim, and Y.-J. Park, "Free-positioning wireless power transfer to multiple devices using a planar transmitting coil and switchable impedance matching networks," IEEE Transactions on Microwave Theory and Techniques, vol. 64, no. 11, pp. 3714-3722, 2016.

[13] X. Huang, Y. Gao, J. Zhou, J. Ma, J. Zhang, and Y. Fang, "Magnetic field design for optimal wireless power transfer to multiple receivers," IET Power Electronics, vol. 9, no. 9, pp. 18851893, 2016.

[14] S. Kisseleff, I. F. Akyildiz, and W. H. Gerstacker, "Magnetic induction-based simultaneous wireless information and power transfer for single information and multiple power receivers," IEEE Transactions on Communications, vol. 65, no. 3, pp. 13961410, 2017.

[15] K. E. Koh, T. C. Beh, T. Imura, and Y. Hori, "Impedance matching and power division using impedance inverter for wireless power transfer via magnetic resonant coupling," IEEE Transactions on Industry Applications, vol. 50, no. 3, pp. 20612070, 2014.

[16] E. Waffenschmidt, "Dynamic resonant matching method for a wireless power transmission receiver," IEEE Transactions on Power Electronics, vol. 30, no. 11, pp. 6070-6077, 2015.

[17] G. Monti, L. Corchia, L. Tarricone, and M. Mongiardo, "A network approach for wireless resonant energy links using relay resonators," IEEE Transactions on Microwave Theory and Techniques, vol. 64, no. 10, pp. 3271-3279, 2016.

[18] W. X. Zhong, C. Zhang, X. Liu, and S. Y. R. Hui, "A methodology for making a three-coil wireless power transfer system more energy efficient than a two-coil counterpart for extended transfer distance," IEEE Transactions on Power Electronics, vol. 30, no. 2, pp. 933-942, 2015.

[19] M. Fu, T. Zhang, X. Zhu, P. C.-K. Luk, and C. Ma, "Compensation of cross coupling in multiple-receiver wireless power transfer systems," IEEE Transactions on Industrial Informatics, vol. 12, no. 2, pp. 474-482, 2016. 


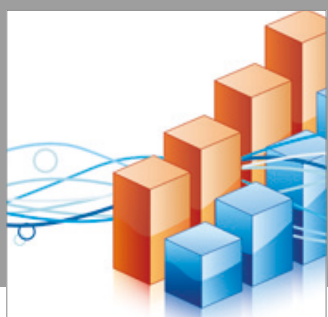

Advances in

Operations Research

vatersals

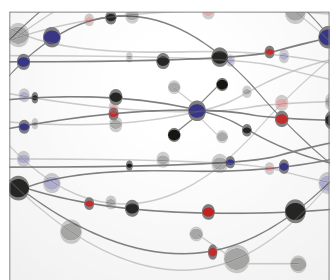

\section{The Scientific} World Journal
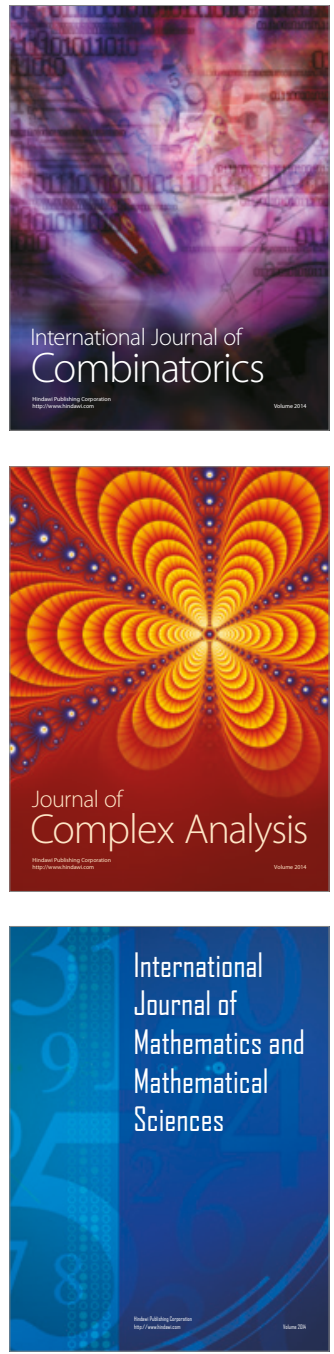
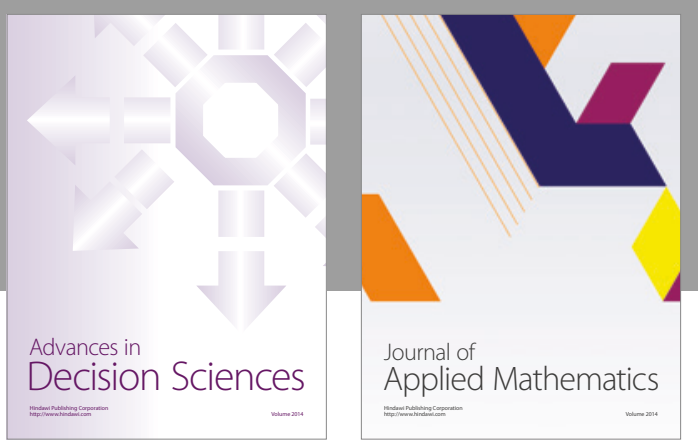

Algebra

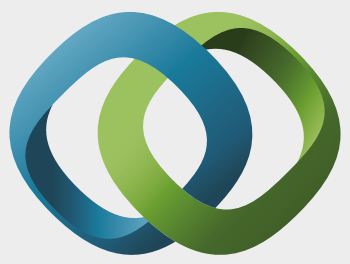

\section{Hindawi}

Submit your manuscripts at

https://www.hindawi.com
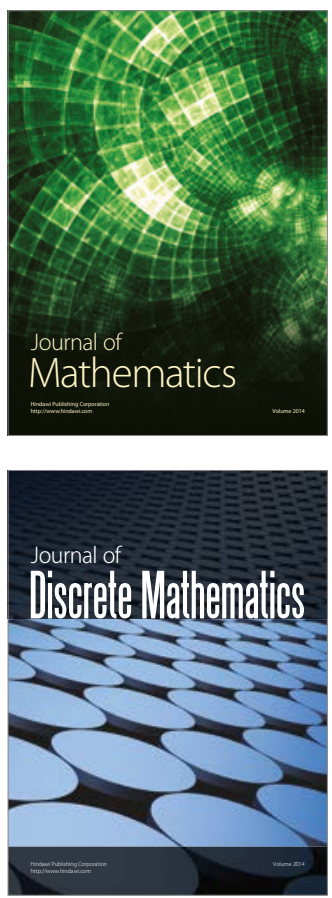

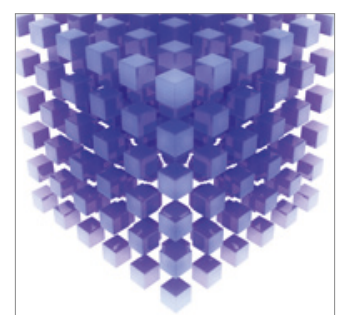

Mathematical Problems in Engineering
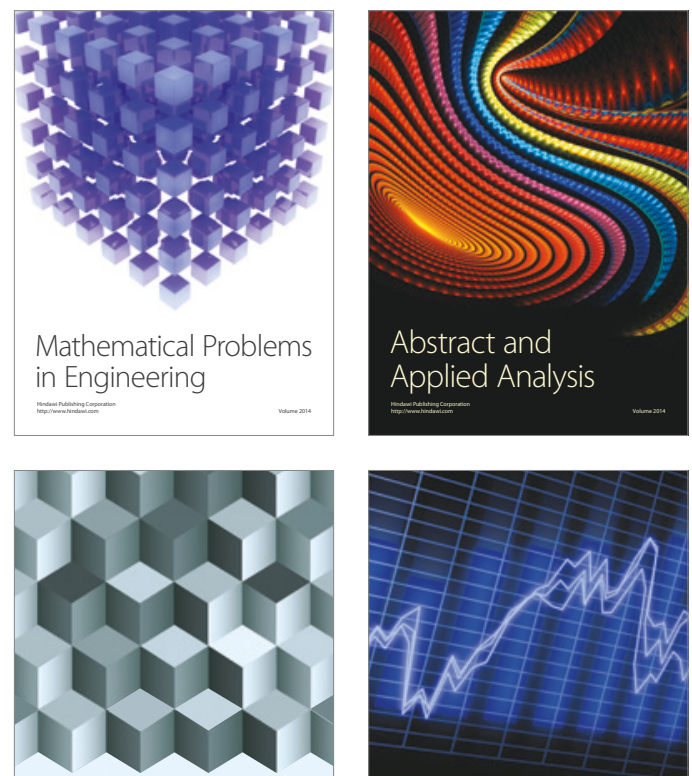

Journal of

Function Spaces

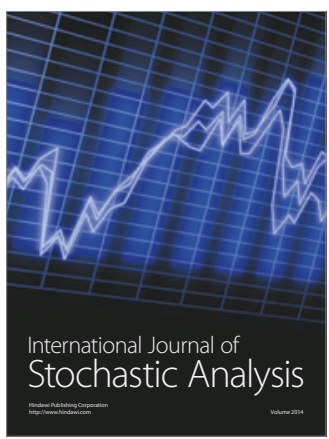

Probability and Statistics
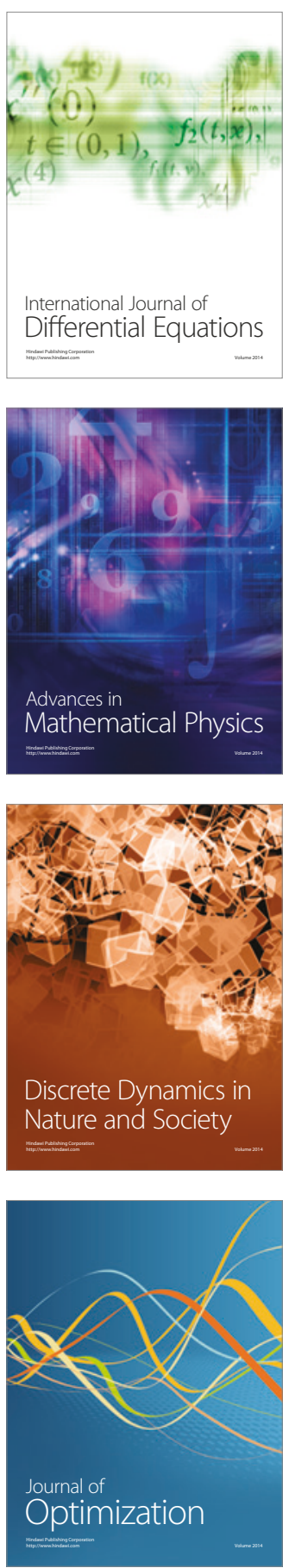